\title{
Rab22a is a novel prognostic marker for cell progression in breast cancer
}

\author{
MIAO HE ${ }^{1 *}$, LEIHUA SHEN ${ }^{2,3 *}$, CHENGWEI JIANG $^{4}$, GE GAO $^{4}$, KEREN WANG $^{3}$, YAN JIAO $^{5}$, \\ LIANG SUN ${ }^{3}$, YINGNAN CUI ${ }^{3}$, ZIRUI KE ${ }^{3,6}$ and ZHAOYING YANG ${ }^{3}$ \\ ${ }^{1}$ Department of Anesthesia, The Second Hospital of Jilin University, Changchun, Jilin 130022; ${ }^{2}$ Department of \\ General Surgery, Xi'an Central Hospital, Xi'an, Shanxi 710000; Departments of ${ }^{3}$ Breast Surgery and ${ }^{4}$ Pathology, \\ China-Japan Union Hospital of Jilin University, Changchun, Jilin 130033; ${ }^{5}$ Department of Hepatobiliary and Pancreatic Surgery, \\ The First Hospital of Jilin University, Changchun, Jilin 130021; ${ }^{6}$ Department of Breast Surgery, Hubei Cancer Hospital, \\ Tongji Medical College, Huazhong University of Science and Technology, Wuhan, Hubei 430000, P.R. China
}

Received July 10, 2019; Accepted January 7, 2020

DOI: $10.3892 /$ ijmm.2020.4486

\begin{abstract}
Breast cancer (BC) is the most common female malignant tumor worldwide. The mechanism of tumorigenesis is still unclear. Ras-related proteins in brain ( $\mathrm{Rab}$ )22a belongs to the Ras superfamily, which may act as an oncogene and participate in carcinogenesis. The present study aims to identify whether Rab22a could be a novel biomarker of prognosis and determine the effects of Rab22a on BC cell progression. A total $258 \mathrm{BC}$ and 56 para-tumor or non-tumor formalin fixed paraffin embedded tissues were stained through immunohistochemistry. The association between Rab22a expression and clinicopathological features, as well as overall survival status were analyzed. The expression level of Rab22a in breast cell lines were detected using reverse transcription-quantitative PCR and western blotting. SK-BR-3 cells were infected with Rab22a short hairpin RNA lenti-virus and the ability of cell
\end{abstract}

Correspondence to: Dr Zirui Ke, Department of Breast Surgery, Hubei Cancer Hospital, Tongji Medical College, Huazhong University of Science and Technology, 116 Zhuodaoquan South Road, Wuhan, Hubei 430000, P.R. China

E-mail:kzr008@hotmail.com

Dr Zhaoying Yang, Department of Breast Surgery, China-Japan Union Hospital of Jilin University, 126 Xiantai Street, Changchun, Jilin 130033, P.R. China

E-mail: zhaoyingyang@163.com

*Contributed equally

Abbreviations: $\mathrm{BC}$, breast cancer; Rab, Ras-related proteins in brain; ER, estrogen receptor; $\mathrm{PR}$, progesterone receptor; Her-2, human epidermal growth factor-2; OS, overall survival; IHC, immunohistochemistry; SC, scramble control; GEO, Gene Expression Omnibus; TCGA, The Cancer Genome Atlas; GSEA, Gene Set Enrichment Analysis; NES, normalized enrichment score; FDR, false discovery rate

Key words: Rab22a, prognosis, progression, breast cancer proliferation, migration and invasion were measured. Gene Set Enrichment Analysis (GSEA) was employed to analyze the pathways involved in the Rab22a mRNA high level group. Rab22a was found to be overexpressed in BC tissues and upregulated in BC cells. High expression of Rab22a was related to a poor prognosis of patients with BC. Knockdown of Rab22a decreased the proliferation, migration and invasion ability of BC cells. GSEA indicated that certain pathways, including mammalian target of rapamycin complex 1 and protein secretion were upregulated, while pathways, such as hypoxia and KRas were downregulated in the Rab22a high level group. Rab22a is of prognostic value for BC and necessary for BC cell proliferation.

\section{Introduction}

Breast cancer $(\mathrm{BC})$ is the most prevalent malignant tumor among females worldwide. It is the most common cancer in China, as well as the United States (1). Fan et al (2) reported that Chinese patients account for over $12 \%$ of all newly diagnosed cases as well as $9 \%$ of all deaths from BC globally. Similarly, Waks and Winer (3) reported that $>250,000$ new patients were diagnosed in the United States in 2017 and they estimated that $12 \%$ of women over the course of their lifetimes will be diagnosed with BC. Depending on levels of estrogen or progesterone receptors (ER and $\mathrm{PR}$, respectively), human epidermal growth factor-2 (Her-2), as well as Ki-67, BC is classified into four major molecular subtypes: Luminal A, Luminal B, Her-2 positive and basal-like. Although the diagnosis, as well as treatment of BC has achieved significant advances and overall survival (OS) has improved during recent decades, there are still challenges regarding the early detection and prediction of survival in BC. Moreover, the mechanism of tumorigenesis is still unclear. Thus, it is important to develop novel biomarkers for diagnosis and prognosis, as well as treatment targets.

The authors' previous study reported that Rab22a is the target gene of microRNAs (miRNAs/miR)-193b in BC (4). Studies of miRNAs have reported that miR-520b (5), miR-204 (6) and miR-203 (7) are downregulated in BC or other types of tumors. In addition, one of the potential targeting 
genes of the above-mentioned miRNAs is Rab22a, which was confirmed using a luciferase assay and is upregulated in these tumor tissues or cell lines. All these findings lead to the conclusion that Rab22a may act as an oncogene and participate in carcinogenesis. Furthermore, emerging evidence suggests that dysregulated expression of Rab22a affects tumor growth. He et al (8) found that Rab22a is upregulated in most of the 11 hepatocellular carcinomas, as well as 1 case of cholangiohepatoma. Okamoto et al (9) identified that Rab22a is positively expressed in malignant melanoma cell lines and situated at the areas of chromosomal breakpoints.

The coding gene of Rab22a, which belongs to the Ras superfamily, is located at chromosome 20q13.32. Rab22a is a part of the Ras-related proteins in brain (Rabs) GTPase subfamily, which are small GTP-binding proteins. Zhou et al (10) reported that Rab22a participates at several levels of the endocytic pathway. In addition, the composition of the plasma membrane receptors is regulated by endocytic recycling and endocytic uptake. Stenmark concluded that Rab22a mediates trafficking among trans-Golgi network and early endosomes (11). Kauppi et al (12) reported that the overexpression of Rab22a in vitro leads to the enlargement of early endosomes. Previous studies have also indicated that the recycling of the transferrin receptor and major histocompatibility complex-I (MHC)-I by endocytosed cargos is blocked by small interfering RNAs that inhibit the function of Rab22a $(13,14)$. It is widely reported that dysregulation of endocytosis is the hallmark of several cancers. Therefore, the abnormal expression of Rab GTPases may be involved in tumorigenesis.

Wang et al (15) reported that the upregulation of Rab22a mRNA in the primary tumor is related to worsening of overall as well as metastasis-free survival, by analyzing The Cancer Genome Atlas (TCGA) database of patients with BC. An additional study found that knockdown of Rab22a inhibits BC metastasis in vivo (15). Kang et al (16) designed and synthesized O(2)-3-Aminopropyl diazeniumdiolates 3a-f, which prevents the growth and metastasis of triple negative $\mathrm{BC}$ in vivo as well as in vitro by MHC-I impairing microvesicle formation by altering the expression of $\mathrm{miR}-203 / \mathrm{Rab} 22 \mathrm{a}$ in a nitric oxide-dependent manner.

Although much of the research indicates that the Rab22a gene can function as an oncogene in several cancer models and indicates its potential therapy target $(15,16)$, whether Rab22a may be developed as a novel prognostic biomarker in $\mathrm{BC}$ is not well understood. The current study applied immunohistochemistry (IHC) staining and analyzed the association between Rab22a expression and clinicopathological features, as well as OS status. The impact of Rab22a on the progression of BC cells as well as the probable pathways involved was also studied.

\section{Materials and methods}

Patients and samples. A total of 258 samples from female patients with BC aged from 27 to 78, who had accepted modified radical mastectomy or breast-conserving surgery at the China-Japan Union Hospital of Jilin University from January 2012 to December 2013 were included in the present study. Patient characteristics are shown in Table I. The clinical stage was distinguished using the guidelines of the American Joint Committee on Cancer. The patients who accepted chemotherapy or radiotherapy and hormone therapy prior to surgery were excluded. Recurrent or metastasis cases at the time of diagnosis were eliminated as well. The last date of follow-up was in April 2019. A total of 56 para-tumor or non-tumor tissues were acquired as the controls. The current study was conducted after obtaining written informed consent from all patients and was approved by the Research Ethics Committee of the China-Japan Union Hospital of Jilin University (approval no. 2019022603).

Breast cell lines. The human mammary gland epithelium cell line, MCF-10A was cultured in a DMED/HAM F12 1:1 mixed medium (Hyclone; GE Healthcare), which contains 5\% horse serum (Gibco; Thermo Fisher Scientific, Inc.), $0.5 \mathrm{mg} / \mathrm{ml}$ hydrocortisone (Sigma-Aldrich; Merck KGaA), $100 \mathrm{ng} / \mathrm{ml}$ cholera toxin (Sigma-Aldrich; Merck KGaA), $10 \mu \mathrm{g} / \mathrm{ml}$ insulin (Sigma-Aldrich; Merck KGaA) and $20 \mathrm{ng} / \mathrm{ml}$ EFG (Peprotech, Inc.). The human BC cell lines, MCF-7 as well as SK-BR-3, were cultured in DMEM H21 (Hyclone; GE Healthcare) and RPMI-1640 (Hyclone; GE Healthcare), each supplemented with $10 \%$ fetal bovine serum (Gibco; Thermo Fisher Scientific, Inc.), $100 \mathrm{U} / \mathrm{ml}$ penicillin and $100 \mathrm{mg} / \mathrm{ml}$ streptomycin. All cell lines were bought from American Type Culture Collection and cultured at $37^{\circ} \mathrm{C}$ with $5 \% \mathrm{CO}_{2}$, in a humidified atmosphere.

IHC staining. In order to identify Rab22a expression levels in the BC tissues, IHC staining was performed by following the manufacturer's protocol (Metal enhanced DAB for IHC; Invitrogen; Thermo Fisher Scientific, Inc.). In brief, after fixation with formalin at room temperature for $48 \mathrm{~h}$ and embedded with paraffin, $3 \mu \mathrm{m}$ thick paraffin sections adhered to slides were incubated at $65^{\circ} \mathrm{C}$ for $30 \mathrm{~min}$, deparaffinized using xylene and then rehydrated using alcohol. The slides were submerged in EDTA for antigen retrieval. Next, the sections were treated with $3 \%$ hydrogen, heated in a microwave at $100^{\circ} \mathrm{C}$ for $5 \mathrm{~min}$ and incubated in $1 \%$ bovine serum albumin (Gibco; Thermo Fisher Scientific, Inc.). Following this, they were incubated with a Rab22a antibody (cat. no. 12125-1-AP; Proteintech, Inc.) at dilution of 1:100 overnight at $4^{\circ} \mathrm{C}$. Normal goat serum served as the negative control. After washing with PBS+1\% Tween, the sections were incubated with the secondary antibody with streptavidin-horseradish peroxidase complex (cat. no. 31460; Invitrogen; Thermo Fisher Scientific, Inc.) at a dilution of 1:2,000 at room temperature for $1 \mathrm{~h}$, followed by colorimetric detection using metal enhanced DAB (cat. no. 34065; Invitrogen; Thermo Fisher Scientific, Inc.) at room temperature for $5 \mathrm{~min}$. All sections were submerged in 3-amino-9-ethyl carbazole, counterstained using 10\% Mayer's hematoxylin at room temperature for $2 \mathrm{~min}$, dehydrated and mounted.

The intensity of IHC staining of the sections was evaluated using a light microscope at x100 magnification by two senior pathologists independently, who were not aware of the patient information and histopathological details. The plasma staining of Rab22a was positive. The staining intensity as well as positive staining percentage of tumor cells were used for evaluation. The intensity of staining was categorized 
Table I. Clinicopathological characteristics of patient samples and expression of Rab22a in breast cancer.

\begin{tabular}{lll}
\hline & \multicolumn{2}{c}{ Rab22a expression } \\
\cline { 2 - 3 } Characteristics & Low Middle High P-value \\
\hline
\end{tabular}

Age, years
$<50$
$\geq 50$

Menopause stage

$\begin{array}{lllll}\text { No } & 20 & 68 & 55 & \\ \text { Yes } & 10 & 57 & 48 & 0.415\end{array}$

Age at menarche,

years

$<15$

$\geq 15$

Clinical stage

I
II
III

T classification

T1
T2
T3
T4

$\mathrm{N}$ classification

N0
N1
N2
N3

Nipple invasion

No

Yes

Venous invasion

No

Yes

Nerve invasion

No

Yes

Basal invasion

No

Yes

Pectoralis minor

muscle invasion

No

Yes

30

124

101

Pathological

differentiation

G1

G2

G3

ER status

Negative

Positive

$\begin{array}{lll}20 & 58 & 45\end{array}$

$\begin{array}{lll}10 & 67 & 58\end{array}$

0.079

0.415

0.001

0.148

0.013

0.640
Low expression

High expression

Molecular classification

Luminal A

Luminal B (Her-2-)

Luminal B (Her-2+)

Her-2+

Basal like

Vital status (at follow-up)

$\begin{array}{lrrrr}\text { Alive } & 28 & 112 & 772 & \\ \text { Dead } & 2 & 13 & 26 & 0.003\end{array}$

T, tumor; M, metastasis; N, node; ER, estrogen receptor; PR, progesterone receptor; Her-2, human epidermal growth factor-2.

as previously described (17): 0 (stainless), 1 (light yellow), 2 (yellow brown) and 3 (brown). The proportion of positive staining tumor cells were $0(0 \%), 1$ (1-10\%), 2 (11-25\%), $3(25-50 \%)$ and $4(>50 \%)$. The score was calculated as staining intensity $\mathrm{x}$ proportion of positive cells and was used as the staining index to calculate IHC intensity (range from 0 to 12). A staining index score of 0 was defined as no expression of Rab22 (-), 1-3 signified low expression of Rab22 (1+), 4-6 signified mild expression of Rab22a (2+), while >6 signified high expression of Rab22a (3+).

Reverse transcription-quantitative polymerase chain reaction (RT-qPCR). Total RNA extraction was done using TRIzol (Ambion; Thermo Fisher Scientific, Inc.). The reverse transcription of total RNA was done using 5X PrimeScript RT Master Mix for Real Time (Takara Bio, Inc.). RT-qPCR was performed using SYBR ${ }^{\circledR}$ Premix DimerEraser ${ }^{\mathrm{TM}}$ Perfect Real Time (Takara Bio, Inc.). The operation process was conducted following the manufacturer's protocol. RT-qPCR analyses were conducted by Mastercycler (Eppendorf). The thermocycling procedure was as follows: $30 \mathrm{sec}$ at $95^{\circ} \mathrm{C}$, followed by 40 cycles of $95^{\circ} \mathrm{C}$ for $30 \mathrm{sec}, 57^{\circ} \mathrm{C}$ for $30 \mathrm{sec}$ and a final step of $68^{\circ} \mathrm{C}$ for $30 \mathrm{~min}$. The primer sequences used were as follows: Rab22a F: 5'-GTCCCTTAGCACCAA TGTACTATC-3', R: 5'-AATGGCAACTACAATATTAGG TGG-3'; GAPDH F: 5'-GAAGGCTGGGGCTCATTTGCA GGG-3', R: 5'-GGTGCAGGAGGCATTGCTGATGAT-3'. The comparative fold-change at mRNA level was calculated 
using the $2^{-\Delta \Delta \mathrm{Cq}}$ method (18). GAPDH served as a reference control for normalization.

Short hairpin (sh)RNA knockdown. The lenti-virus of Rab22a shRNA was purchased from Hanheng Biotechnology to target human Rab22a. The SK-BR-3 cells were infected with Rab22a shRNA or a scramble control (SC) lenti-virus as per the supplier's protocol. In brief, $2 \times 10^{5}$ SK-BR-3 cells were incubated with $2 \times 10^{7}$ transducing units of lenti-virus and $8 \mu \mathrm{g}$ polybrene (Hanheng Biotechnology) in $1 \mathrm{ml}$ serum-free medium at $37^{\circ} \mathrm{C}$ with $5 \% \mathrm{CO}_{2}$ for $8 \mathrm{~h}$. Consequently, $1 \mathrm{ml}$ fresh growth medium was added for $24 \mathrm{~h}$ and was changed with fresh growth media. Stable cell lines were generated in the selected medium with $0.5 \mu \mathrm{g} / \mathrm{ml}$ puromycin after $48 \mathrm{~h}$ of infection.

Western blotting. The cells were harvested in Lysis Buffer for the western blot analysis and IP (cat. no. P0013; Shanghai Biyuntian Bio-Technology Co., Ltd.) and the proteins were extracted. The Bio-Rad Laboratories, Inc., method was applied to quantify protein concentration. Proteins $(25 \mu \mathrm{g} /$ well) were separated on a $10 \%$ SDS-PAGE gel and then transferred onto a nitrocellulose membrane. After blocking with $5 \%$ fat-free milk at room temperature for $1 \mathrm{~h}$, the membranes were incubated with Rab22a (cat. no. 12125-1-AP; Proteintech, Inc.) or GAPDH antibodies (cat. no. 60004-1-Ig, Proteintech, Inc.) at a dilution of 1:1,000, overnight at $4^{\circ} \mathrm{C}$. Anti-rabbit secondary antibodies were conjugated with horseradish peroxidase (cat. no. SA00001; Proteintech, Inc.) at room temperature for $1 \mathrm{~h}$. Immunoreactivity was detected using enhanced chemiluminescence (BeyoECL Plus; Shanghai Biyuntian Bio-Technology Co., Ltd.) and visualized through autoradiography.

Cell proliferation assay. Cell proliferation abilities were tested using Cell Counting Kit-8 assays (CCK-8; Dalian Meilunbio Biotechnology Co., Ltd.) according to the manufacturer's protocol. A total of 3,500 cells/well (SK-BR-3 SC and shRNA) were suspended in 96-well plates in serum free medium, overnight. Once the cells were adhered to the plate, serum free medium was discarded and $100 \mu 1$ growth medium was added into all wells. After incubation for 24, 48, 72 and $96 \mathrm{~h}, 10 \mu \mathrm{l}$ CCK- 8 was added into each well and cultured for $4 \mathrm{~h}$. The optical density value was read at $450 \mathrm{~nm}$ using a microplate reader (BioTek China).

Migration and invasion assay. Cell migration and invasion assays were conducted using BD BioCoat Matrigel Invasion Chambers and Control Inserts (BD Biosciences; Becton, Dickinson and Company). A total of $5 \times 10^{4} \mathrm{SC}$ or knockdown of SK-BR-3 cells were re-suspended in $0.5 \mathrm{ml}$ medium with $1 \%$ fetal bovine serum (FBS), then seeded on either upper control inserts or trans-well chambers with Matrigel (BD Biosciences; Becton, Dickinson and Company). A total of $2 \mathrm{ml}$ medium containing 15\% FBS was added onto the lower chamber, which served as a chemo-attractant. A total of $20 \mathrm{~h}$ later, migrating or invading cells that had attached to the lower surface of the membrane insert were fixed at room temperature for $2 \mathrm{~min}$ and stained by Giemsa at room temperature for $10 \mathrm{~min}$, then counted under a light microscope (Olympus IX51; Olympus Corporation) at x200 magnification.

Mice xenograft model. A total of six female nude mice (Beijing Huafukang Bioscience, Co., Ltd.) that weighed 20-25 g, aged 4-5 weeks were separated into two groups. Each mouse was injected with $4 \times 10^{6}$ SK-BR-3 cells (Rab22a SC or shRNA3) into its fourth mammary fat pad. Tumor size was measured using a caliper every 3 days after injection. Tumor volume was calculated as follows: Volume $=\left(\right.$ length $\mathrm{x}$ width $\left.{ }^{2}\right) / 2$. The mice were sacrificed by inhalation of $\mathrm{CO}_{2}$ in $10 \% / \mathrm{min}$, the criteria for confirmation of death is that heart and breath rate were completely stopped, and pupillary response to light was disappeared. Once the xenograft models were established for 30 days, the xenograft tumors were excised and weighed after euthanasia. The mice were housed in the SPF facility, at $22 \pm 2^{\circ} \mathrm{C}$ with a $12 \mathrm{~h}$ light/dark cycle and a relative humidity of $40-60 \%$, and had free access to food and water, following the Laboratory Animal Care and Use guideline of Jilin University. Animal protocol were approved by the Laboratory Animal Care and Use Committee of Jilin University (approval no. 201905005).

Bioinformatics analysis. For this study, Gene Expression Omnibus (GEO) datasets (GSE25487, GSE22796 and GSE18672) were downloaded from the GEO database (19-21) (https://www.ncbi.nlm.nih.gov/geo/). The expression difference between discrete variables was visualized using the ggplot2 package of $\mathrm{R}$ software (version 3.5.2; http://www.R-project.org/bioconductor.org/), by constructing boxplots. Gene set enrichment analysis (GSEA) was done using GSEA software 3.0. First, the mRNA files consisting of 1,104 BC tumors and 114 normal tissues were downloaded from TCGA database. The high and low mRNA group were defined as the median value of mRNA level of Rab22a. Then, GSEA was performed as previously described (22-24). In brief, the annotated gene sets of 'h.all.v6.2.symbols.gmt', which summarize and represent specific, biological states or processes, for use with GSEA software were acquired from the Molecular Signatures Database (MSigDB). The normalized enrichment score (NES) was determined by investigating permutations for 1,000 times. A $\mathrm{P}<0.05$ as well as false discovery rate $(\mathrm{FDR})<0.25$ were considered to signify the statistical significance of an enriched gene set.

Statistical analysis. SPSS software version 21.0 (IBM Corps.) was used for analysis. Analysis among expression of Rab22a and clinicopathological characteristics of patients were carried out using the chi-square test or Fisher's exact test. Bivariate correlations among study variables were assessed using Spearman's correlation analysis. Survival curves were constructed with Kaplan-Meier plots and compared using the Log-rank test. Multivariate Cox-regressive survival analysis was estimated by including all the parameters that were significant for the univariate Cox-regressive analysis. Data among or between groups were investigated by one-way analysis of variance (ANOVA) or Student's t-test, respectively. LSD (3 groups) and Tukey's (4 groups) post hoc tests were used following ANOVA. $\mathrm{P}<0.05$ was considered to indicate a statistically significant difference. All experiments were conducted in triplicate and the outcomes are presented as the mean \pm standard deviation. 

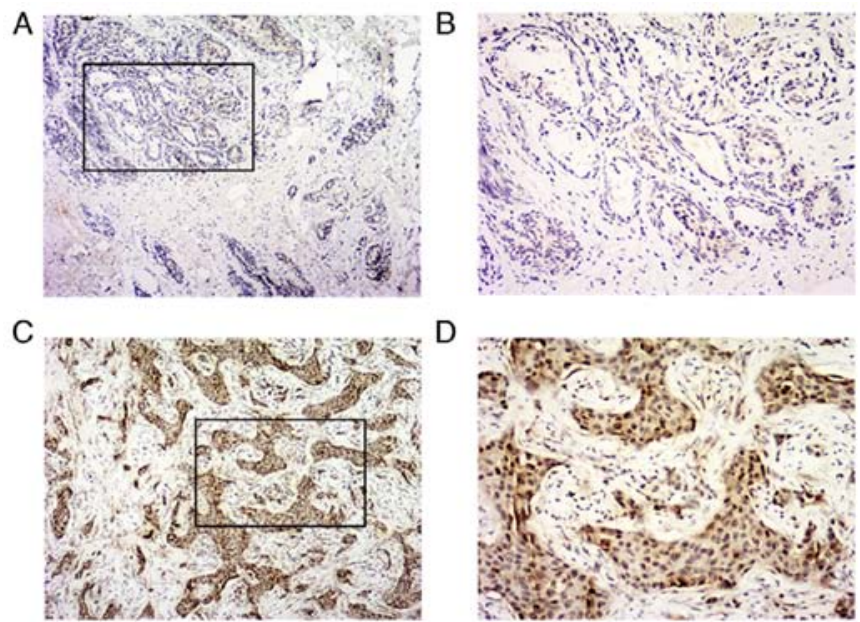

E
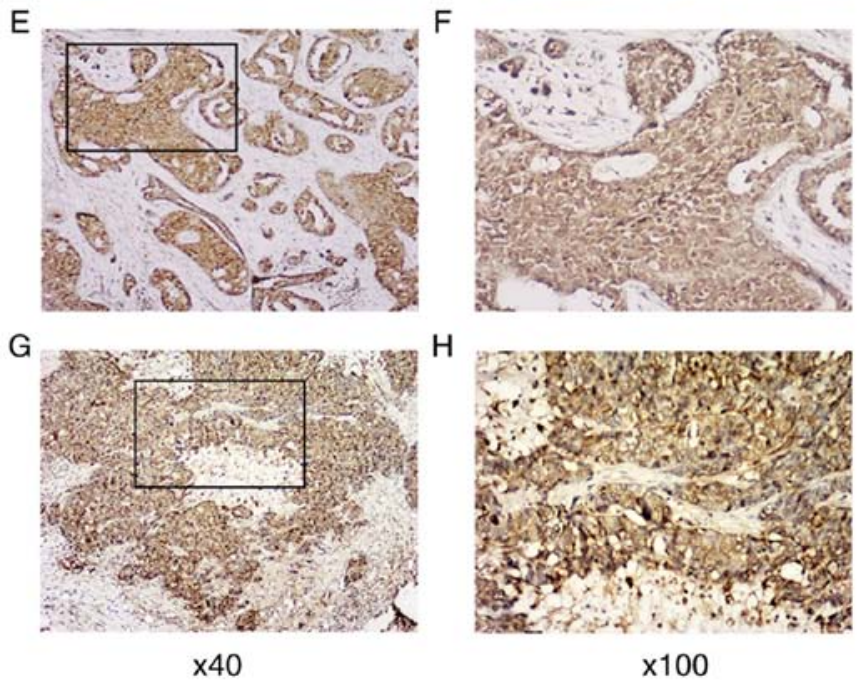

$\mathrm{H}$

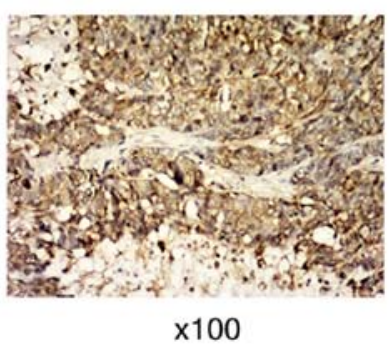

Figure 1. Representative immunochemistry staining images of Rab22a expression in normal breast tissues and breast cancer tissues. (A) Normal breast tissue at low and (B) high magnification. (C) Low and (D) high magnification of tissues with low expression of Rab22a. (E) Mild expression of Rab22a at low and (F) high magnification. $(\mathrm{G})$ Low and $(\mathrm{H})$ high magnification of high expression of Rab22a. Images on the right column (x100) are the magnification of square area of images in the right column (x40). Rab, Ras-related proteins in brain.

\section{Results}

Rab22a is overexpressed in BC tissues. In order to examine the status of Rab22a expression in BC, its expression was inspected by IHC staining of 258 paraffin-embedded BC tissues, containing 98 cases of clinical stage I BC, 98 cases of clinical stage II BC and 62 cases of clinical stage III BC, and compared them with 56 non-tumor or para-tumor samples. The clinical and pathological characteristics of all $258 \mathrm{BC}$ patients are summarized in Table I. Compared with the 56 cases of nearby non-tumor breast tissues, in which Rab22a is untraceable or is expressed at low levels, Rab22a was found to be overexpressed in BC tissues. Rab22a expression in cancer tissues was successfully identified in 3 levels, including low expression (1+) in 30 cases, mild expression (2+) in 125 cases and high expression (3+) in 103 cases (Fig. 1). GEO datasets were employed to verify Rab22a expression. Rab22a expression was significantly increased in tumor samples, compared with normal samples (Fig. 2A and B). Furthermore, Rab22a

Table II. Spearman analysis of correlation between Rab22a and clinicopathological features.

Rab22a expression level

\begin{tabular}{lcc}
\cline { 2 - 3 } Characteristics & Spearman correlation & P-value \\
\hline Age & 0.118 & 0.058 \\
Menopause stage & 0.058 & 0.356 \\
Age at menarche & 0.191 & 0.002 \\
Clinical stage & 0.199 & 0.001 \\
T classification & 0.171 & 0.006 \\
N classification & 0.165 & 0.008 \\
Nipple invasion & 0.151 & 0.015 \\
Venous invasion & 0.154 & 0.014 \\
Nerve invasion & 0.032 & 0.608 \\
Basal invasion & 0.063 & 0.310 \\
Pectoralis minor muscle & 0.063 & 0.310 \\
invasion & & \\
Pathologic differentiation & 0.066 & 0.288 \\
ER status & -0.016 & 0.090 \\
PR status & -0.112 & 0.073 \\
Expression of Her-2 & 0.129 & 0.038 \\
Expression of Ki67 & 0.104 & 0.095 \\
Molecular classification & 0.170 & 0.006 \\
Vital states & -0.171 & 0.006 \\
\hline
\end{tabular}

T, tumor; M, metastasis; N, node; ER, estrogen receptor; PR, progesterone receptor; Her-2, human epidermal growth factor-2.

expression was significantly increased in all four molecular subtypes (Luminal A and B, Her-2 and basal-like) compared with normal tissues (Fig. 2C).

Rab22a overexpression is correlated with the clinicopathological features of $B C$. The connection between expression of Rab22a and clinicopathological characteristics of patients with BC was analyzed (Table I). Rab22a expression was found to be related with age at menarche $(\mathrm{P}=0.005)$, clinical stage ( $\mathrm{P}=0.001)$, N classification $(\mathrm{P}=0.013)$, nipple invasion $(\mathrm{P}=0.042)$, venous invasion $(\mathrm{P}=0.044)$ and vital states $(\mathrm{P}=0.003)$. There was no difference based on age, menopause stage, $\mathrm{T}$ classification, nerve invasion, basal invasion, pectoralis minor muscle invasion, pathological differentiation, ER status, PR status, expression of Her-2, expression of Ki67 and molecular classification among the levels of Rab22a expression.

Spearman analysis of correlation among Rab22a and clinicopathological features indicate that the expression of Rab22a is significantly positively associated with age at menarche $(\mathrm{P}=0.002)$, clinical stage $(\mathrm{P}=0.001)$, $\mathrm{T}$ classification $(\mathrm{P}=0.006)$, $\mathrm{N}$ classification $(\mathrm{P}=0.008)$, nipple invasion $(\mathrm{P}=0.015)$, venous invasion $(\mathrm{P}=0.014)$, expression of Her-2 $(\mathrm{P}=0.038)$ and molecular classification $(\mathrm{P}=0.006)$, while negatively associated with vital states $(\mathrm{P}=0.006)$ (Table II).

High Rab22a expression is associated with the poor prognosis of patients with BC. In the Kaplan-Meier estimation, 
Table III. Univariate and multivariate Cox-regression analysis of various prognostic parameters in patients with breast cancer.

\begin{tabular}{|c|c|c|c|c|c|c|}
\hline \multirow[b]{2}{*}{ Characteristics } & \multicolumn{3}{|c|}{ Univariate analysis } & \multicolumn{3}{|c|}{ Multivariate analysis } \\
\hline & HR & $95 \% \mathrm{CI}$ & P-value & HR & $95 \% \mathrm{CI}$ & P-value \\
\hline Age at menarche & 0.958 & $0.804-1.142$ & 0.634 & & & \\
\hline Rab22a & 2.285 & $1.325-3.942$ & 0.003 & 2.351 & $1.338-4.132$ & 0.003 \\
\hline Clinical stage & 1.300 & $0.844-2.004$ & 0.234 & & & \\
\hline $\mathrm{T}$ classification & 0.997 & $0.609-1.630$ & 0.989 & & & \\
\hline $\mathrm{N}$ classification & 1.320 & $0.986-1.768$ & 0.062 & & & \\
\hline Expression of Her-2 & 1.326 & $1.008-1.745$ & 0.044 & 1.282 & $0.958-1.714$ & 0.094 \\
\hline Molecular classification & 1.647 & $0.925-2.930$ & 0.090 & & & \\
\hline
\end{tabular}

HR, hazard ratio; CI, confidence interval; T, tumor; N, node; Her-2, human epidermal growth factor-2.
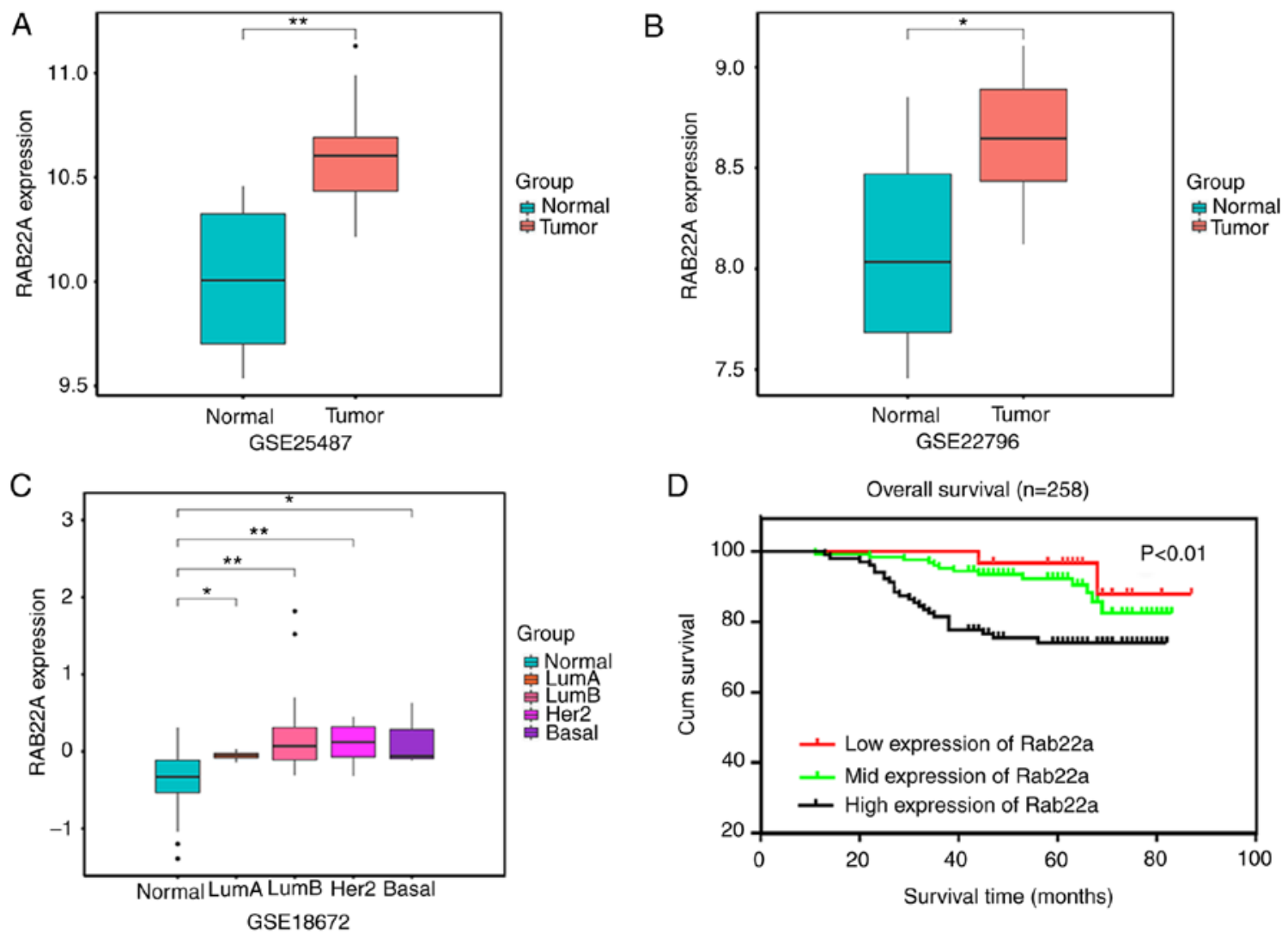

Figure 2. GEO dataset verifies Rab22a expression and overall survival. Rab22a expression in GEO databases (A) GSE25487 and (B) GSE22796 (normal vs. tumor); (C) Rab22a expression in GEO database (normal vs. molecular subtypes of breast cancer). (D) Overall survival curve of low, middle and high expression of Rab22a in patients with breast cancer $(\mathrm{n}=258)$ plotted as given by Kaplan-Meier analysis. ${ }^{*} \mathrm{P}<0.05$ and ${ }^{* *} \mathrm{P}<0.01$. GEO, Gene Expression Omnibus; Rab, Ras-related proteins in brain.

the median OS time in patients with BC was 94.1 weeks for those with low expression of Rab22a, 93.7 weeks for mild expression of Rab22a and 81.2 weeks for high expression of Rab22a ( $<<0.01)$ (Fig. 2D). This indicates that patients with high expression of Rab22a had poorer OS.

Univariate and multivariate Cox-regression analysis were conducted to identify factors related with OS of patients with BC (Table III). The univariate Cox-regression analysis indicates that a high level of expression of Rab22a $(\mathrm{P}=0.003)$ and Her-2 $(\mathrm{P}=0.044)$ were related to poor OS. Then, a high level of expression of $\operatorname{Rab} 22 \mathrm{a}(\mathrm{P}=0.003)$ was identified to be associated with short OS in the multivariate Cox-regression analysis. These results infer that Rab22a could be an independent predictor for $\mathrm{BC}$.

Rab22a is upregulated in BC cell lines and required for cell progression. mRNAs were isolated from MCF-10A (control) and BC cell lines MCF-7, as well as SK-BR-3, and examined using RT-qPCR. The mRNA level of Rab22a in MCF-7 and SK-BR-3 was 23 times greater than that of 

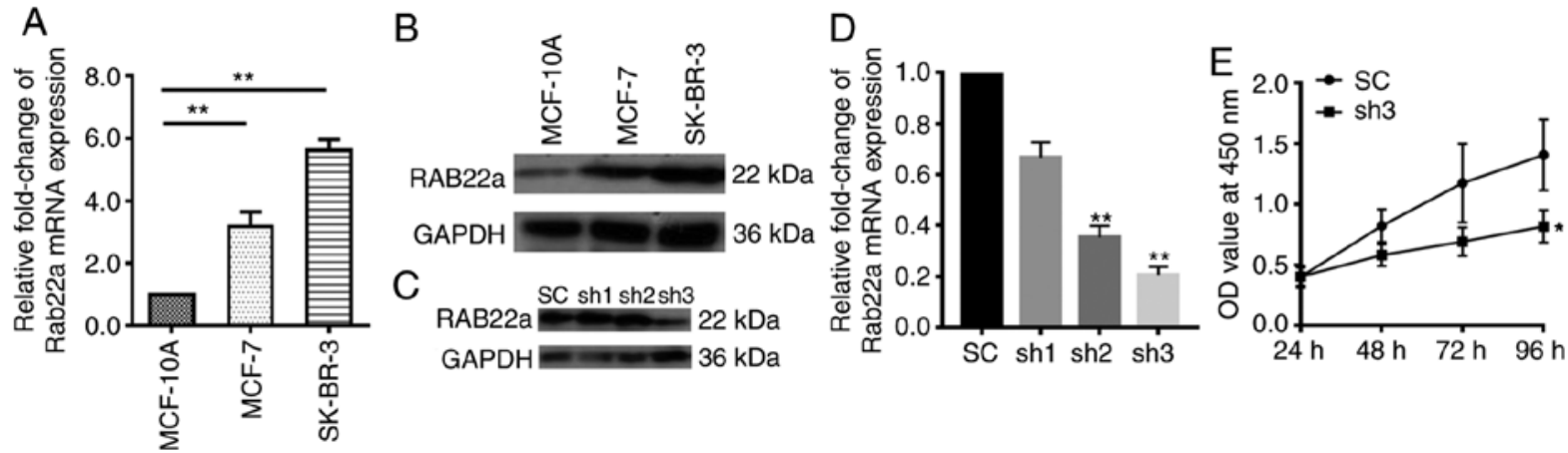

Figure 3. Rab22a is upregulated in breast cancer cell lines and necessary for cell proliferation. (A) mRNA expression and (B) protein levels of Rab22a in MCF-10A, MCF-7 and SK-BR-3 cells were detected using reverse transcription-quantitative PCR and western blotting. The mean Rab22a mRNA expression was normalized to the expression of GAPDH. (C) Rab22a protein and (D) mRNA levels in SK-BR-3 cells after being infected with a SC or Rab22a shRNA lent-virus. (E) The ability of proliferation of SK-BR-3 cells decreased in Rab22a shRNA knockdown cells (sh), compared with SC. A total of three independent experiments were done for each assay. ${ }^{*} \mathrm{P}<0.05$ and ${ }^{* *} \mathrm{P}<0.001$ vs. the SC group. SC, scramble control; sh, short hairpin; Rab, Ras-related proteins in brain.
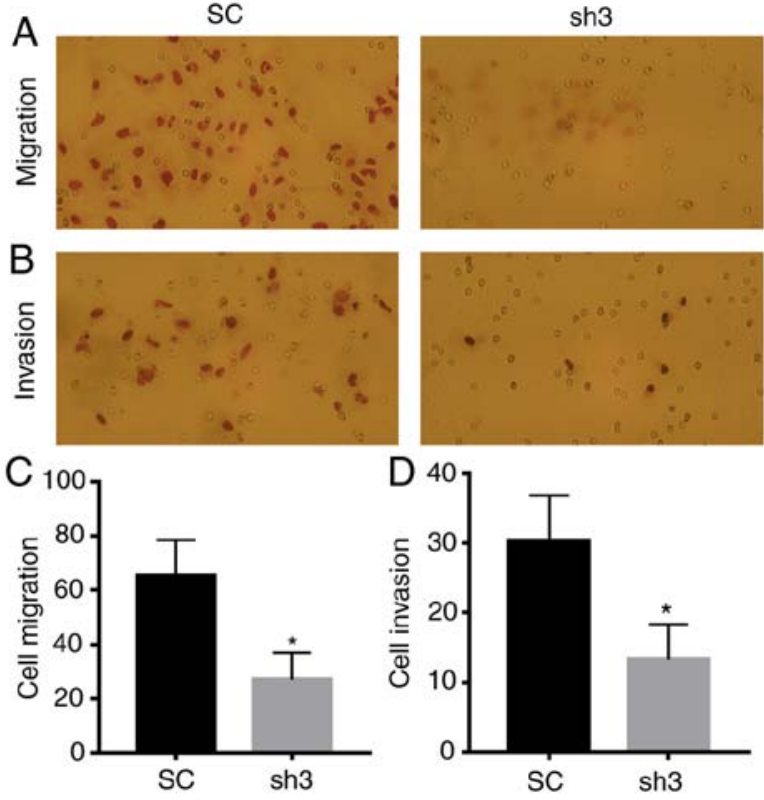

Figure 4. Knockdown of Rab22a decrease migration and invasion of BC cells. Downregulated Rab22a levels inhibit the (A) migration and (B) invasion capabilities of $\mathrm{BC}$ cells (magnification $\mathrm{x} 200$ ) and quantitative analysis of cell (C) migration and (D) invasion. ${ }^{*} \mathrm{P}<0.05$ vs. SC. SC, scramble control; sh, short hairpin; Rab, Ras-related proteins in brain.

MCF-10A ( $\mathrm{P}<0.001$; Fig. 3A). The results were confirmed at the protein level using western blotting analysis (Fig. 3B). After a stable Rab22a shRNA knockdown cell line was established in SK-BR-3 cells, mRNA and protein levels of Rab22a were examined using RT-qPCR and western blotting, respectively (Fig. 3C and D). The mRNA level of Rab22a in SK-BR-3 shRNA2 and shRNA3 was $70 \%$ lower than that of the SK-BR-3 SC $(\mathrm{P}<0.001)$. The proliferation of SK-BR-3 shRNA and SK-BR-3 SC cells were examined continuously for $96 \mathrm{~h}$. It was found that Rab22a-shRNA showed a significantly lower proliferation rate than SK-BR-3 $\mathrm{SC}$ cells $(\mathrm{P}<0.05$; Fig. 3E). Moreover, downregulation of Rab22a significantly inhibited the migration and invasion of BC cells $(\mathrm{P}<0.05$; Fig. 4).

The xenograft mice model showed that the tumor weight of Rab22a SC and knockdown group was $0.86 \pm 0.11 \mathrm{~g}$ vs.
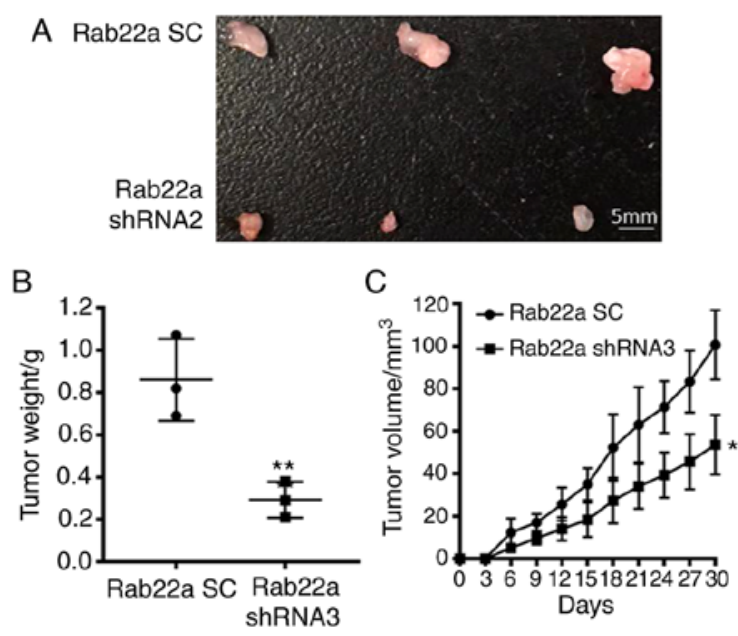

Figure 5. Rab22a is necessary for tumor growth in vivo. (A) Xenograft tumors were excised and (B) weighed at the end-point. (C) Tumor volume was measured every 3 days during 30 day observation period. ${ }^{*} \mathrm{P}<0.05$ and ${ }^{* *} \mathrm{P}<0.01$ vs. SC group. SC, scramble control; sh, short hairpin; Rab, Ras-related proteins in brain.

$0.29 \pm 0.05 \mathrm{~g}(\mathrm{P}<0.01$; Fig. 5A and $\mathrm{B})$ and tumor volume was $100.70 \pm 9.40 \mathrm{~mm}^{3}$ vs. $53.67 \pm 8.09 \mathrm{~mm}^{3}$ (P<0.05; Fig. 5C) at the endpoint date. The results of the in vitro and in vivo experiments indicate that the proliferation ability of $\mathrm{BC}$ is significantly inhibited in Rab22a knockdown group, compared with the SC group.

GSEA identifies the Rab22a-related signaling pathways. In order to recognize signaling cascades related with Rab22a in $\mathrm{BC}, \mathrm{GSEA}$ was employed to analyze pathways differentiated in the Rab22a high and low expressed data sets. GSEA indicated noteworthy changes $(\mathrm{FDR}<0.25, \mathrm{P}<0.05)$ in the enrichment of the MSigDB Collection. The most significantly enriched signaling cascades based on NES values are presented in Fig. 6. GSEA found that in the Rab22a overexpression group, 'E2F targets', 'G2M checkpoint', 'mammalian target of rapamycin complex 1 (mTorc1) pathway', 'protein secretion' and 'myc targets pathways' are upregulated (Fig. 6A-E), while 'hypoxia', 'KRas signal-down', 'tumor necrosis factor (TNF)- $\alpha$ signal via nuclear factor $(\mathrm{NF}) \kappa \mathrm{B}$ and p53 pathways' are downregulated (Fig. 6F-I). 

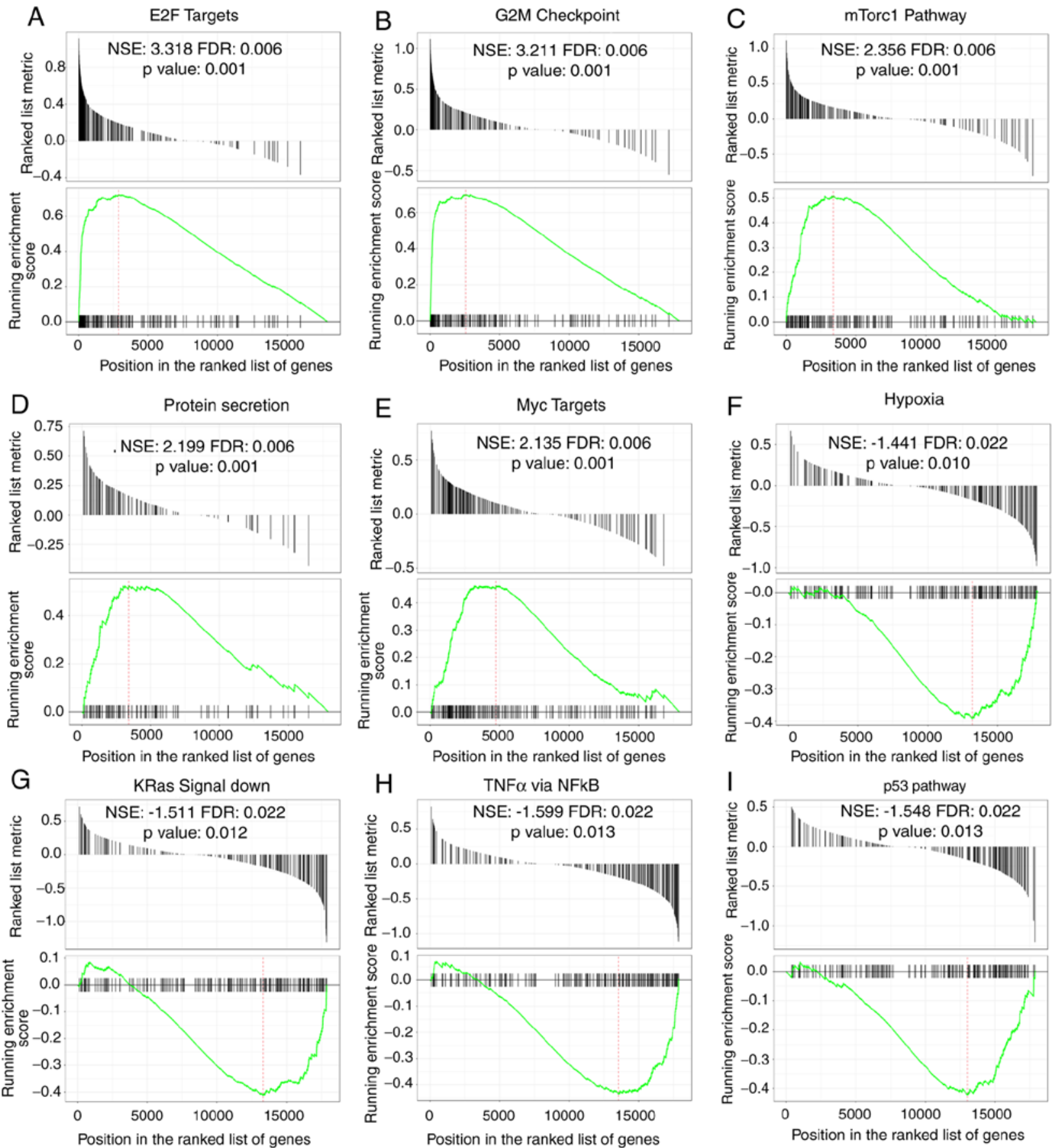

Figure 6. GSEA analysis revealed the pathways involved in the Rab22a high expression group. E2F targets (A) G2M checkpoint (B) mTorc1 pathway (C) protein secretion (D) myc targets pathways (E) are upregulated, while hypoxia (F) KRas signal down (G) TNF- $\alpha$ signal via NFKB (H) p53 pathways (I) are downregulated. NES, normalized enrichment score; FDR, false discovery rate; GSEA, Gene Set Enrichment Analysis; TNF, tumor necrosis factor; mTorc1, mammalian target of rapamycin complex 1 ; NF, nuclear factor.

\section{Discussion}

A number of studies on the possible function of Rab22a have emerged during recent years $(25,26)$. However, the role of Rab22a in $\mathrm{BC}$ has not been discussed as much. In the present study, the correlation between Rab22a expression and clinicopathological characters and patient OS were examined. It was found that Rab22a is overexpressed in BC tissues and that high expression of Rab22a is an independent prognostic factor of $\mathrm{BC}$. Moreover, Rab22a was found to be required for the proliferation, migration and invasion of $\mathrm{BC}$ cells.
Rab22a is a member of the RAS oncogene family and the protein encoded gene is a member of the RAB family of small GTPases. At present, >60 Rab members have been recognized (27). The function of Rab proteins is regulated by the cycling of the proteins between GTP-bound active and GDP-bound inactive states, with the relative amount of these two small GTPase states regulated by GTPase-activating proteins and guanine nucleotide exchange factors (28). Rab GTPases are considered the main regulators of secretory and endocytic pathways (29). Endocytosis plays an imperative role in cellular processes, including nutrient uptake and receptor signaling (30). 
Some Rab GTPases function as membrane trafficking regulators and determine protein presentation for the neoplastic cell membrane (31). Defective vesicular trafficking of growth factor receptors has been found to be a manifold hallmark of cancerous cells (32). Rab22a has been reported to control the trafficking of recycling endosomes comprising clathrin-dependent factors, such as epidermal growth factor receptor, transferrin receptor protein 2, copper-transporting ATPase 1 and glucose transporter 4, as well as clathrin-independent factors, such as MHC-I, $\beta 2$-adrenergic receptor and integrin b1 receptor are endocytosed cargoes to the cell surface in non-melanocytes (26).

Rab22a is dysregulated in numerous cancer types. However, the prognostic value of Rab22a has not been previously investigated in $\mathrm{BC}$. The present study found that Rab22a expression is related to clinical stage, $\mathrm{N}$ classification, nipple invasion, venous invasion and vital state. Increased expression of Rab22a in the present BC cohorts is positively related to a poor prognosis in patients. In the current study, it was shown that $\mathrm{Rab} 22 \mathrm{a}$ is an independent prognosis factor of BC. Therefore, Rab22a may be a novel survival predictive biomarker for BC. These findings suggest that the overexpression of Rab22a may act as a possible biomarker to identify $\mathrm{BC}$ that may result in a poorer clinical outcome.

In order to investigate whether Rab22a affects $\mathrm{BC}$ progression, Rab22a mRNA and protein levels were found to be upregulated in $\mathrm{BC}$ cells compared with normal epithelium cells. Knockdown of Rab22a inhibits BC cell proliferation in vitro and in vivo. Moreover, loss function of Rab22a expression decreases the migration and invasion capabilities of SK-BR-3 cells. The present findings are consistent with other research reports. Zhou et al (10) reported that Rab22a is overexpressed in lung cancer and that knockdown of Rab22a represses the migration and invasion of lung cancer cells. Moreover, Rab22a improves recycling of cluster of differentiation (CD)147 that is necessary for lung cancer cell migration and invasion. A further study performed by Qi et al (33) identified that the transmembrane protein, YIPY2, promotes CD147-medated malignant phenotypes by recruiting and activating Rab5 and Rab22a in hepatocellular carcinoma. Su et al (17) found that the increased expression of $\mathrm{Rab} 22 \mathrm{a}$ is related to an advanced clinical stage in melanoma and shorter OS. The present study suggests that Rab22a may act as a novel oncogene in $\mathrm{BC}$ and may be a potential therapeutic target.

Atypical and uncontrolled cell proliferation is a hallmark of malignancy and is triggered by the dysregulation of the expression of numerous vital proteins. Although emerging evidence shows the regulation between Rab22a and non-coding RNAs in different types of tumors $(7,34)$, the regulatory mechanism of transcription or the signaling pathway of Rab22a is not well studied. Therefore, a GSEA analysis was performed to study the potential pathways related with Rab22a. GSEA analyses indicated that in the Rab22a overexpression group, 'E2F targets', 'G2M checkpoint', 'mTorc1 pathway', 'protein secretion' and 'myc target pathways' are upregulated, while 'hypoxia', 'KRas signal-down', 'TNF- $\alpha$ signal via NFKB and p53 pathways' are downregulated. A set of Rab proteins have been reported to promote tumor aggression by regulating intracellular signaling. The signaling pathway identified by GSEA is consistent with that of other published studies (35-37) Overexpression of Rab1A promotes colorectal cancer progression and invasion by activating mTorcl signaling. Moreover, it has been shown that $\mathrm{BC}$ stem cell development is promoted by Rab2A through activation of extracellular signaling (38). While low Rab1B expression is reportedly correlated with poor prognosis of $\mathrm{BC}$, downregulation of Rab1B was found to promote triple-negative breast cancer (TNBC) metastasis by activating transforming growth factor- $\beta /$ mothers against decapentaplegic homolog signaling (39). Hypoxia-inducible factors induces microvesicle (MV) generation by upregulating Rab22a expression in human BC cells. However, Rab22a knockdown abrogates MV generation under hypoxic conditions and impairs lung metastasis (40). However, the detailed underlying mechanism still requires additional investigation. One limitation of the present research is the loss-function study was only performed in one cell line; further studies including gain-function will be conducted in multiple BC cell lines.

In conclusion, this study has shown that Rab22a is overexpressed in human BC tissues and cell lines, and is a prognostic biomarker that is required for cell progression. The present research suggests that Rab22a may be regarded as a possible biomarker of prognosis and treatment of $\mathrm{BC}$ in the future.

\section{Acknowledgements}

Not applicable.

\section{Funding}

The present study was supported by the Science and Technology of Jilin Province Health and Family Planning Commission Project 2017Q035(ZYY).

\section{Availability of data and materials}

All data generated or analyzed during this study are included in this published article.

\section{Authors' contributions}

$\mathrm{MH}$ wrote the manuscript, and performed the data analysis and interpretation. LS performed the statistical analysis and followed-up the patients. CJ, ZK and GG performed the immunochemistry staining estimation. KW collected the samples. YJ performed the Gene Set Enrichment Analysis. LS, and YC performed the in vivo experiments. ZK performed the in vitro experiments. ZY designed the study. All authors read and approved the final manuscript.

\section{Ethics approval and consent to participate}

This study was approved by the Research Ethics Committee of the China-Japan Union Hospital of Jilin University (grant no. 2019022603). Animal protocol was approved by the Laboratory Animal Care and Use Committee of Jilin University (grant no. 201905005). This study was conducted with written informed consent given by all patients.

\section{Patient consent for publication}

This study was conducted with written informed consent from all patients. 


\section{Competing interest}

The authors declare that there are no conflicts of interest.

\section{References}

1. DeSantis CE, Ma J, Gaudet MM, Newman LA, Miller KD, Goding Sauer A, Goding Sauer A, Jemal A and Siegel RL: Breast cancer statistics, 2019. CA Cancer J Clin 69: 438-451, 2019.

2. Fan L, Strasser-Weippl K, Li JJ, St Louis J, Finkelstein DM, Yu KD, Chen WQ, Shao ZM and Goss PE: Breast cancer in China. Lancet Oncol 15: e279-e289, 2014.

3. Waks AG and Winer EP: Breast cancer treatment: A review. JAMA 321: 288-300, 2019.

4. Sun L, He M, Xu N, Xu DH, Ben-David Y, Yang ZY and Li YJ: Regulation of RAB22A by mir-193b inhibits breast cancer growth and metastasis mediated by exosomes. Int J Oncol 53: 2705-2714, 2018

5. Zhang L and Yu S: Role of miR-520b in non-small cell lung cancer. Exp Ther Med 16: 3987-3995, 2018.

6. Xiong F, Liu K, Zhang F, Sha K, Wang X, Guo X and Huang N: miR-204 inhibits the proliferation and invasion of renal cell carcinoma by inhibiting RAB22A expression. Oncol Rep 35: 3000-3008, 2016.

7. Yang D, Liu G and Wang K: miR-203 acts as a tumor suppressor gene in osteosarcoma by regulating RAB22A. PLoS One 10 e0132225, 2015

8. He H, Dai F, Yu L, She X, Zhao Y, Jiang J, Chen X and Zhao S: Identification and characterization of nine novel human small GTPases showing variable expressions in liver cancer tissues. Gene Expr 10: 231-242, 2002.

9. Okamoto I, Pirker C, Bilban M, Berger W, Losert D, Marosi C, Haas OA, Wolff $\mathrm{K}$ and Pehamberger H: Seven novel and stable translocations associated with oncogenic gene expression in malignant melanoma. Neoplasia 7: 303-311, 2005.

10. Zhou Y, Wu B, Li JH, Nan G, Jiang JL and Chen ZN: Rab22a enhances CD147 recycling and is required for lung cancer cell migration and invasion. Exp Cell Res 357: 9-16, 2017.

11. Stenmark H: Rab GTPases as coordinators of vesicle traffic. Nat Rev Mol Cell Biol 10: 513-525, 2009.

12. Kauppi M, Simonsen A, Bremnes B, Vieira A, Callaghan J, Stenmark $\mathrm{H}$ and Olkkonen VM: The small GTPase Rab22 interacts with EEA1 and controls endosomal membrane trafficking. J Cell Sci 115: 899-911, 2002.

13. Magadan JG, Barbieri MA, Mesa R, Stahl PD and Mayorga LS Rab22a regulates the sorting of transferrin to recycling endosomes. Mol Cell Biol 26: 2595-2614, 2006.

14. Weigert R, Yeung AC, Li J and Donaldson JG: Rab22a regulates the recycling of membrane proteins internalized independently of clathrin. Mol Biol Cell 15: 3758-3770, 2004.

15. Wang T, Gilkes DM, Takano N, Xiang L, Luo W, Bishop CJ, Chaturvedi P, Green JJ and Semenza GL: Hypoxia-inducible factors and RAB22A mediate formation of microvesicles that stimulate breast cancer invasion and metastasis. Proc Natl Acad Sci USA 111: E3234-E3242, 2014

16. Kang F, Zhu J, Wu J, Lv T, Xiang H, Tian J, Zhang Y and Huang Z: O(2)-3-Aminopropyl diazeniumdiolates suppress the progression of highly metastatic triple-negative breast cancer by inhibition of microvesicle formation via nitric oxide-based epigenetic regulation. Chem Sci 9: 6893-6898, 2018.

17. Su F, Chen Y, Zhu S, Li F, Zhao S, Wu L, Chen X and Su J: RAB22A overexpression promotes the tumor growth of melanoma. Oncotarget 7: 71744-71753, 2016.

18. Livak KJ and Schmittgen TD. Analysis of relative gene expression data using real-time quantitative PCR and the 2(-Delta Delta C(T)) method. Methods 25: 402-408, 2001.

19. Bennett CN, Tomlinson CC, Michalowski AM, Chu IM, Luger D, Mittereder LR, Aprelikova O, Shou J, Piwinica-Worms H, Caplen NJ, et al: Cross-species genomic and functional analyses identify a combination therapy using a CHK1 inhibitor and a ribonucleotide reductase inhibitor to treat triple-negative breast cancer. Breast Cancer Res 14: R109, 2012.

20. Tan MH, De S, Bebek G, Orloff MS, Wesolowski R, Downs-Kelly E, Budd GT, Stark GR and Eng C: Specific kinesin expression profiles associated with taxane resistance in basal-like breast cancer. Breast Cancer Res Treat 131: 849-858, 2012.
21. Haakensen VD, Biong M, Lingjaerde OC, Holmen MM, Frantzen JO, Chen Y, Navjord D, Romundstad L, Lüders T, Bukholm IK, et al: Expression levels of uridine 5'-diphospho-glucuronosyltransferase genes in breast tissue from healthy women are associated with mammographic density. Breast Cancer Res 12: R65, 2010.

22. Mootha VK, Lindgren CM, Eriksson KF, Subramanian A, Sihag S, Lehar J, Puigserver P, Carlsson E, Ridderstråle M, Laurila E, et al: PGC-1alpha-responsive genes involved in oxidative phosphorylation are coordinately downregulated in human diabetes. Nat Genet 34: 267-273, 2003.

23. Subramanian A, Tamayo P, Mootha VK, Mukherjee S, Ebert BL, Gillette MA, Paulovich A Pomeroy SL, Golub TR, Lander ES and Mesirov JP: Gene set enrichment analysis: A knowledge-based approach for interpreting genome-wide expression profiles. Proc Natl Acad Sci USA 102: 15545-15550, 2005.

24. Jiao Y, Fu Z, Li Y, Meng L and Liu Y: High EIF2B5 mRNA expression and its prognostic significance in liver cancer: A study based on the TCGA and GEO database. Cancer Manag Res 10: 6003-6014, 2018.

25. Cebrian I, Croce C, Guerrero NA, Blanchard N and Mayorga LS: Rab22a controls MHC-I intracellular trafficking and antigen cross-presentation by dendritic cells. EMBO Rep 17: 1753-1765, 2016.

26. Shakya S, Sharma P, Bhatt AM, Jani RA, Delevoye C and Setty SR: Rab22A recruits BLOC-1 and BLOC-2 to promote the biogenesis of recycling endosomes. EMBO Rep 19: e45918, 2018.

27. Ishibashi $K$, Kanno $E$, Itoh $T$ and Fukuda $M$ : Identification and characterization of a novel Tre-2/Bub2/Cdc16 (TBC) protein that possesses Rab3A-GAP activity. Genes Cells 14: 41-52, 2009.

28. Mesa R, Magadan J, Barbieri A, Lopez C, Stahl PD and Mayorga LS: Overexpression of Rab22a hampers the transport between endosomes and the Golgi apparatus. Exp Cell Res 304: 339-353, 2005

29. Pfeffer SR: Rab GTPases: Master regulators that establish the secretory and endocytic pathways. Mol Biol Cell 28: 712-715, 2017.

30. Maldonado-Baez L and Donaldson JG: Hook1, microtubules, and Rab22: Mediators of selective sorting of clathrin-independent endocytic cargo proteins on endosomes. Bioarchitecture 3 : 141-146, 2013.

31. Goldenring JR: A central role for vesicle trafficking in epithelial neoplasia: Intracellular highways to carcinogenesis. Nat Rev Cancer 13: 813-820, 2013

32. Mosesson Y, Mills GB and Yarden Y: Derailed endocytosis: An emerging feature of cancer. Nat Rev Cancer 8: 835-850, 2008.

33. Qi S, Su L, Li J, Zhao P, Zhang Q, Niu X, Liu J, Jia G, Wei X, Tavernier J, et al: YIPF2 is a novel Rab-GDF that enhances HCC malignant phenotypes by facilitating CD147 endocytic recycle. Cell Death Dis 10: 462, 2019.

34. Zheng S, Jiang F, Ge D, Tang J, Chen H, Yang J, Yao Y, Yan J, Qiu J, Yin Z, et al: LncRNA SNHG3/miRNA-15la-3p/RAB22A axis regulates invasion and migration of osteosarcoma. Biomed Pharmacother 112: 108695, 2019.

35. Mayorga LS and Cebrian I: Rab22a: A novel regulator of immune functions. Mol Immunol 11: 87-92, 2018.

36. Rodriguez-Furlan C, Domozych D, Qian W, Enquist PA, Li X, Zhang C, Schenk R, Winbigler HS, Jackson W, Raikhel NV and Hicks GR: Interaction between VPS35 and RABG3f is necessary as a checkpoint to control fusion of late compartments with the vacuole. Proc Natl Acad Sci USA 116: 21291-21301, 2019.

37. Rajarajacholan UK, Thalappilly S and Riabowol K: The ING1a tumor suppressor regulates endocytosis to induce cellular senescence via the Rb-E2F pathway. PLoS Biol 11: e1001502, 2013.

38. Luo ML, Gong C, Chen $\mathrm{CH}$, Hu H, Huang P, Zheng M, Yao Y, Wei S, Wulf G, Lieberman J, et al: The Rab2A GTPase promotes breast cancer stem cells and tumorigenesis via Erk signaling activation. Cell Rep 11: 111-124, 2015.

39. Jiang HL, Sun HF, Gao SP, Li LD, Hu X, Wu J and Jin W: Loss of RAB1B promotes triple-negative breast cancer metastasis by activating TGF- $\beta /$ SMAD signaling. Oncotarget 6: 16352-16365, 2015.

40. Wu H, Carvalho P and Voeltz GK: Here, there, and everywhere: The importance of ER membrane contact sites. Science 361: 6401,2018

This work is licensed under a Creative Commons Attribution-NonCommercial-NoDerivatives 4.0 International (CC BY-NC-ND 4.0) License. 